\title{
Mathematical Analysis of Nonimaging Fresnel Lenses Using Refractive and Total Internal Reflection Prisms for Sunlight Concentration
}

\author{
Perla M. Viera-González $\mathbb{D}^{\mathbb{D}}{ }^{1}$ Guillermo E. Sánchez-Guerrero, ${ }^{1}$ \\ Edgar Martínez-Guerra, ${ }^{1}$ and Daniel E. Ceballos-Herrera ${ }^{2}$ \\ ${ }^{1}$ Universidad Autónoma de Nuevo León, Facultad de Ciencias Físico Matemáticas, Av. Universidad S/N Ciudad Universitaria, \\ 66455 San Nicolás de los Garza, Mexico \\ ${ }^{2}$ Instituto de Ingeniería, Universidad Nacional Autónoma de México, Cd. Universitaria, 04510 Ciudad de México, Mexico \\ Correspondence should be addressed to Perla M. Viera-González; marlene.viera.gzz@gmail.com
}

Received 30 March 2018; Accepted 31 July 2018; Published 27 September 2018

Academic Editor: Ivan D. Rukhlenko

Copyright @ 2018 Perla M. Viera-González et al. This is an open access article distributed under the Creative Commons Attribution License, which permits unrestricted use, distribution, and reproduction in any medium, provided the original work is properly cited.

During the design of nonimaging Fresnel lenses, the reflectance losses at the refracting surfaces should be taken into account because, depending on the parameters of each prism, semiacceptance angle $\theta_{\text {in }}$ and output angle $\theta_{\text {out }}$, these losses can decrease the total efficiency of the lens. On this subject, an analysis and comparison between a Fresnel lens composed only of refractive prisms and others with total internal reflection (TIR) prisms are presented and, using this information, a design method of hybrid Fresnel lenses composed of refractive and TIR prisms is proposed to reduce reflectance losses from the surface. The work presents the radiance profile and transmittance for a Fresnel lens of each type, to compare its advantages and disadvantages providing an alternative for the design and construction of nonimaging Fresnel lenses according to optical devices requirements.

\section{Introduction}

Fresnel lenses were invented to be used in lighthouses, but over the years their applications have been increased to solar concentration, projectors, copying machines, magnifiers, holography, and communications, among others. The different applications of Fresnel lenses, especially in the field of solar concentration, led to the design of nonimaging Fresnel lenses and the research of new design methods and optimization techniques $[1,2]$.

The imaging Fresnel lenses are designed to have a focal point where all the light is concentrated; in the case of the nonimaging Fresnel lenses, following the design method proposed by Leutz et al., the lens is designed to focus in a limited area, instead of a point. In the next sections, this area is called focal area [1].

As all the optical lenses, imaging and nonimaging Fresnel lenses are prone to different types of losses; some of these losses are as follows [3]: (i) Absorption losses. These are related to the material and the optical path length.

(ii) Chromatic losses. These losses are due to the chromatic dispersion of the light as a result of the changes in the refractive index of the material according to the wavelength of their incident light $(n(\lambda))$. The chromatic aberrations may cause geometrical losses when, as an effect of the chromatic dispersion in the boundary within the range boundaries of wavelengths collected, the light is collected outside the focal area.

(iii) Reflection losses. The light reaching the boundary of 2 materials is not totally refracted; instead, some portion of this light is reflected; these losses are called Fresnel losses.

It is well known that Fresnel losses at the refractive surfaces can be reduced using total internal reflection (TIR) prisms and the chromatic aberration, using the edge ray 
principle adapted to the design method proposed by Leutz [3-5].

This work presents the design method for a nonimaging Fresnel lens composed of a combination of refraction and total internal reflection prisms facing inward to reduce losses by reflection and to avoid chromatic aberrations considering the extreme wavelengths collected [1,3-5]. Besides, a comparison between refractive, total internal reflection and hybrid lenses (composed of refractive and total internal reflection prisms) is presented.

\section{Design Method}

The nonimaging Fresnel lenses are usually dome-shaped lenses integrated by a chain of prisms, and these have an acceptance semiangle $\theta_{i n}$, but it is possible to design nonimaging Fresnel lenses with different shapes. This work is focused on the optimization of dome-shaped nonimaging Fresnel lenses.

These lenses offer advantages as the reduction of focal aberration, lower dependence to sun-tracking mechanisms, and mechanical stability, among others. In contrast, their disadvantages are complications in the manufacturing process, the increment of reflection, and cosine losses [1].

For the design of a nonimaging Fresnel lens it is necessary to design, using an iterative method, each prism considering, as input parameters, the acceptance semiangle $\theta_{\text {in }}$ (this work only covers the nonimaging lenses with only one acceptance semiangle, but, according to Leutz et al., it is possible to design a lens with cross-sectional acceptance semiangle and perpendicular acceptance semiangle [1]), the refractive index $n$ in terms of the wavelength range from its shortest wavelength $\lambda_{s}$, to the highest $\lambda_{h}$, and its focal distance $f$. The present work takes this approach as the start point and includes a process of analyzing which kind of prism, refractive or TIR, has the bigger transmittance for increasing the total transmittance of the whole lens.

2.1. Refractive Prism Design Method. The conventional nonimaging Fresnel lenses consist of a chain of refractive prisms, whose optical path is described in Figure 1, where it includes each one of the incidence angles, through a refraction prism [1].

Using the Snell law and considering the optical path in Figure 1, the prism can be designed following the next equations [1]:

$$
\begin{aligned}
\phi_{0} & =\beta-\alpha+\theta_{i n}, \\
\phi_{1} & =\arcsin \frac{\sin \phi_{0}}{n}, \\
\phi_{2} & =\beta-\phi_{1}, \\
\phi_{3} & =\arcsin \left(n \times \sin \phi_{2}\right), \\
\theta_{\text {out }} & =\phi_{3}-\alpha,
\end{aligned}
$$

where the angles $\theta_{\text {in }}, \phi_{0}, \phi_{1}, \phi_{2}, \phi_{3}, \alpha$, and $\theta_{\text {out }}$ are shown in Figure 1 . The prism angle $\beta$ can not be solved analytically

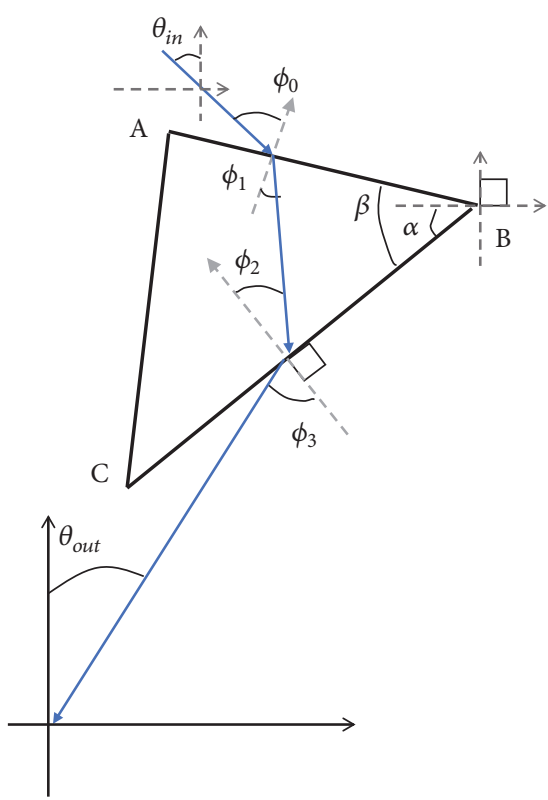

FIGURE 1: Optical path through a refraction prism delimited by the points $\mathrm{A}, \mathrm{B}$, and $\mathrm{C}$.

from (1)-(5); therefore the prism inclination angle $\alpha$ must be determined using approximation methods and considering a margin error $\Delta E$, usually defined as $\Delta E=10^{-5}[1]$, that allows the convergence in the solution of $\beta$ for the cases where $\theta_{\text {in }}<0^{\circ}$ and $\theta_{\text {in }}>0^{\circ}$. Finally, the $3 \mathrm{D}$ shape of each lens is obtained applying rotational symmetry over the optical axis of the resulting profile (Figure 2).

2.2. Chromatic Aberration. To avoid the chromatic aberration in nonimaging Fresnel lenses, the edge-ray principle can be modified taking the following into account $[1,5]$ :

(1) The upper and lower wavelength to collect, where each wavelength is corresponding to different refractive indexes.

(2) The longest wavelength is coming at $+\theta_{i n}$, and it is assigned to the edge of the right-hand side.

(3) The shortest wavelength is coming at $-\theta_{i n}$, and it is assigned to the edge of the left-hand side.

With the steps above, the design method allows that the complete wavelength range can be collected inside the same area (Figure 3). It is important to use an equation that relates the refractive index and wavelength of light for a particular transparent material; one approximation of this relation is described by Cauchy's equation $[1,6]$. Then, the refractive indexes, $n_{s}$ and $n_{h}$, are defined by the shortest and longest wavelengths $\lambda_{s}$ and $\lambda_{h}$.

2.3. Transmittance. The amount of light reflected at the refracting surfaces depends on the angle of incidence and the polarization of the radiation. For the case of sunlight, it is considered nonpolarized; thus, parallel and perpendicular 


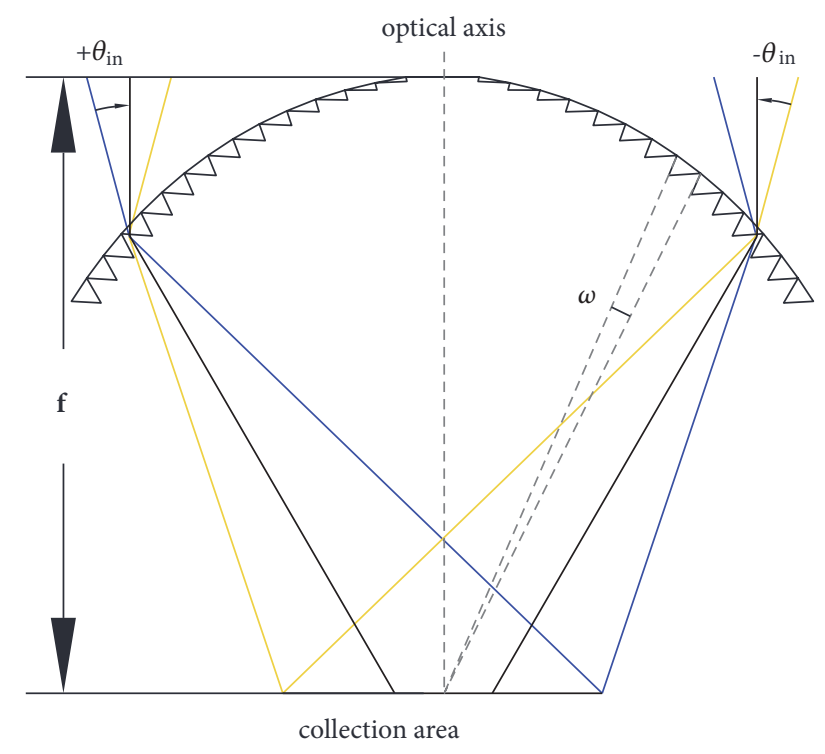

FIGURE 2: Solving (1)-(5) in each prism, the resulting profile is a chain of prisms describing a dome-shaped lens. The angle $\omega$ is defined as the aperture angle of each prism.

reflections have been calculated using the Fresnel equations and can be obtained for each prism as follows $[1,3]$ :

$$
\begin{aligned}
\tau_{\text {total }}= & \left(1-\frac{1}{2}\left(\frac{\tan ^{2}\left(\phi_{1}-\phi_{0}\right)}{\tan ^{2}\left(\phi_{1}+\phi_{0}\right)}+\frac{\sin ^{2}\left(\phi_{1}-\phi_{0}\right)}{\sin ^{2}\left(\phi_{1}+\phi_{0}\right)}\right)\right) \\
& \cdot\left(1-\frac{1}{2}\left(\frac{\tan ^{2}\left(\phi_{3}-\phi_{2}\right)}{\tan ^{2}\left(\phi_{3}+\phi_{2}\right)}+\frac{\sin ^{2}\left(\phi_{3}-\phi_{2}\right)}{\sin ^{2}\left(\phi_{3}+\phi_{2}\right)}\right)\right)
\end{aligned}
$$

where the angles $\phi_{0}, \phi_{1}, \phi_{2}$, and $\phi_{3}$ are shown in Figure 1 and can be solved using (1) to (5). The final value of $\tau_{\text {total }}$ will vary according to the numeric solutions of $\phi_{0}, \phi_{1}, \phi_{2}$, and $\phi_{3}$ that are dependable on the value of $\theta_{i n}$.

2.4. Total Internal Reflection Prisms Design. One of the disadvantages of using nonimaging Fresnel lenses is the losses at the refractive surfaces [1]; to reduce that, the present work proposes the use of total internal reflection (TIR) prism like the one shown in Figure 4. This prism is proposed as a right triangle with the angle $B$ as the right angle. This is proposed because the use of prisms with TIR allows the reduction of the angles $\phi_{2}$ and $\phi_{3}$, which are the angles that increase its value when its distance from the center of the lens is increased.

The optical path inside TIR prism follows the next equations:

$$
\begin{aligned}
\phi_{0} & =\alpha+\theta_{i n}, \\
\phi_{1} & =\arcsin \frac{\sin \phi_{0}}{n}, \\
\phi_{2} & =\beta-\phi_{1}, \\
\phi_{3} & =\arcsin \left(n \times \sin \phi_{2}\right), \\
\theta_{\text {out }} & =\phi_{3}-\alpha,
\end{aligned}
$$

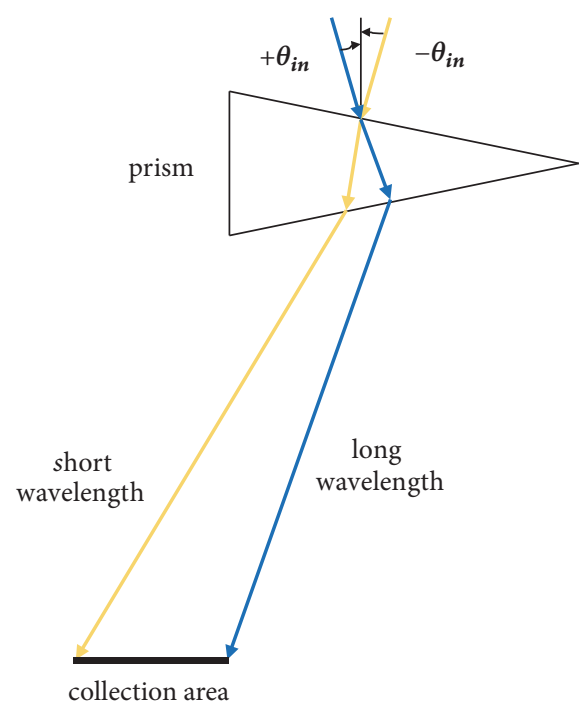

FIGURE 3: Prism design method taking chromatic aberration into account.

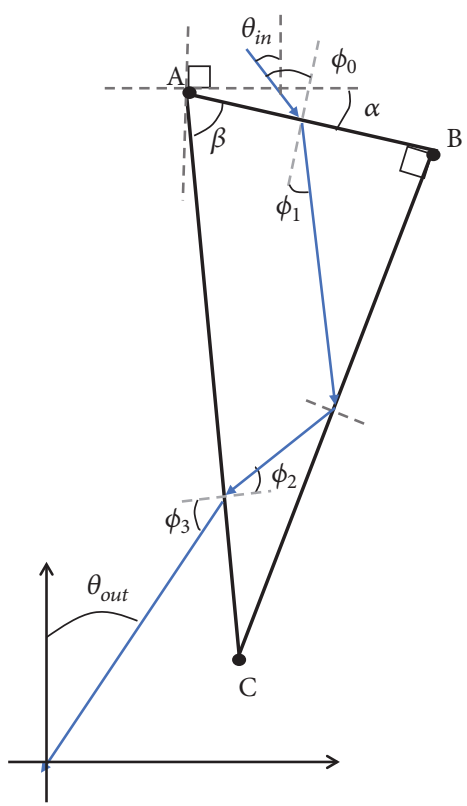

FIGURE 4: The optical path inside a total internal reflection prism delimited by the points A, B, and C.

where the refractive index $n$ will be defined as $n_{s}$ and $n_{h}$ taking into account the extreme values of the wavelengths according to the equation (this equation is used considering Polymethyl Methacrylate (PMMA) as the material of the lens):

$$
n=1.4779+\frac{5.0496 \times 10^{5}}{\lambda^{2}}-\frac{6.9486 \times 10^{11}}{\lambda^{4}}
$$

where the wavelength $\lambda$ is measured in $\AA$ and angles $\theta_{i n}, \phi_{0}$, $\phi_{1}, \phi_{2}, \phi_{3}, \alpha$, and $\theta_{\text {out }}$ are shown in Figure 4 . The construction of each TIR prism must be performed by an iterative method just as the case of the refractive prism and following the Leutz method, but considering the new equations above. 


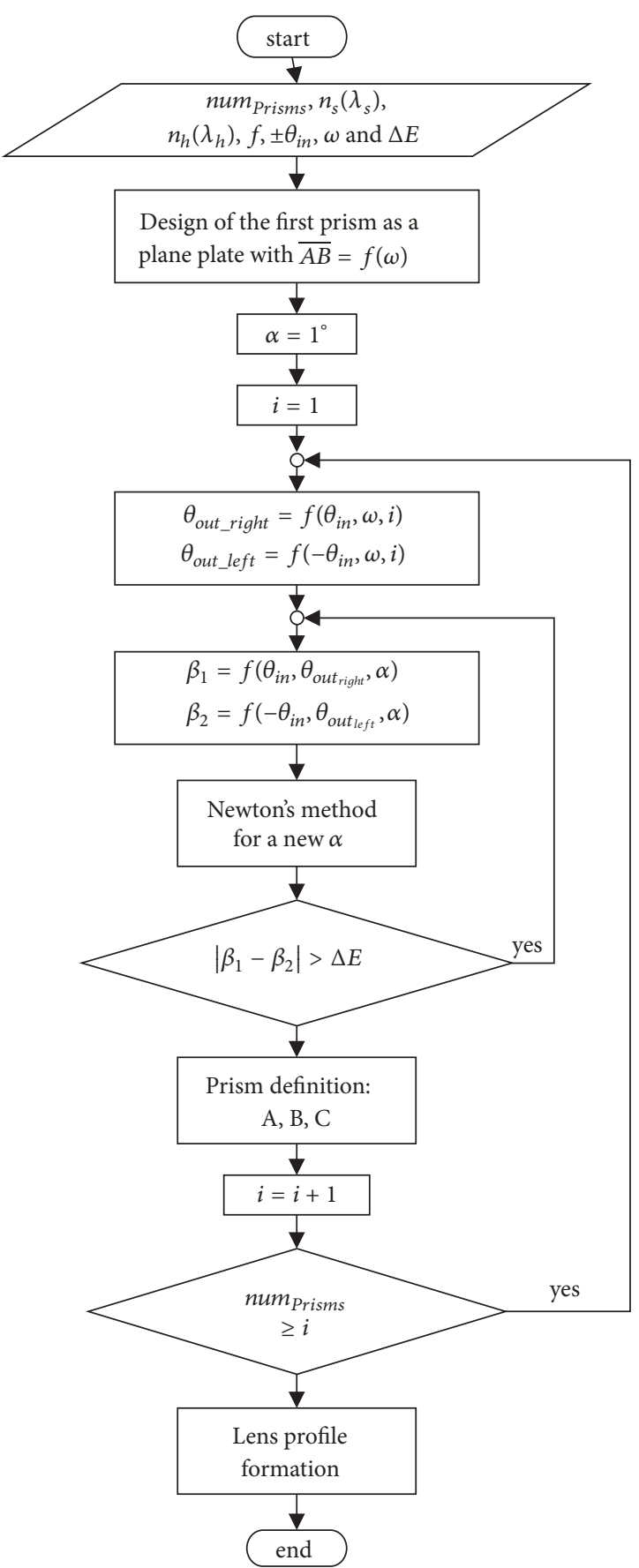

(a)

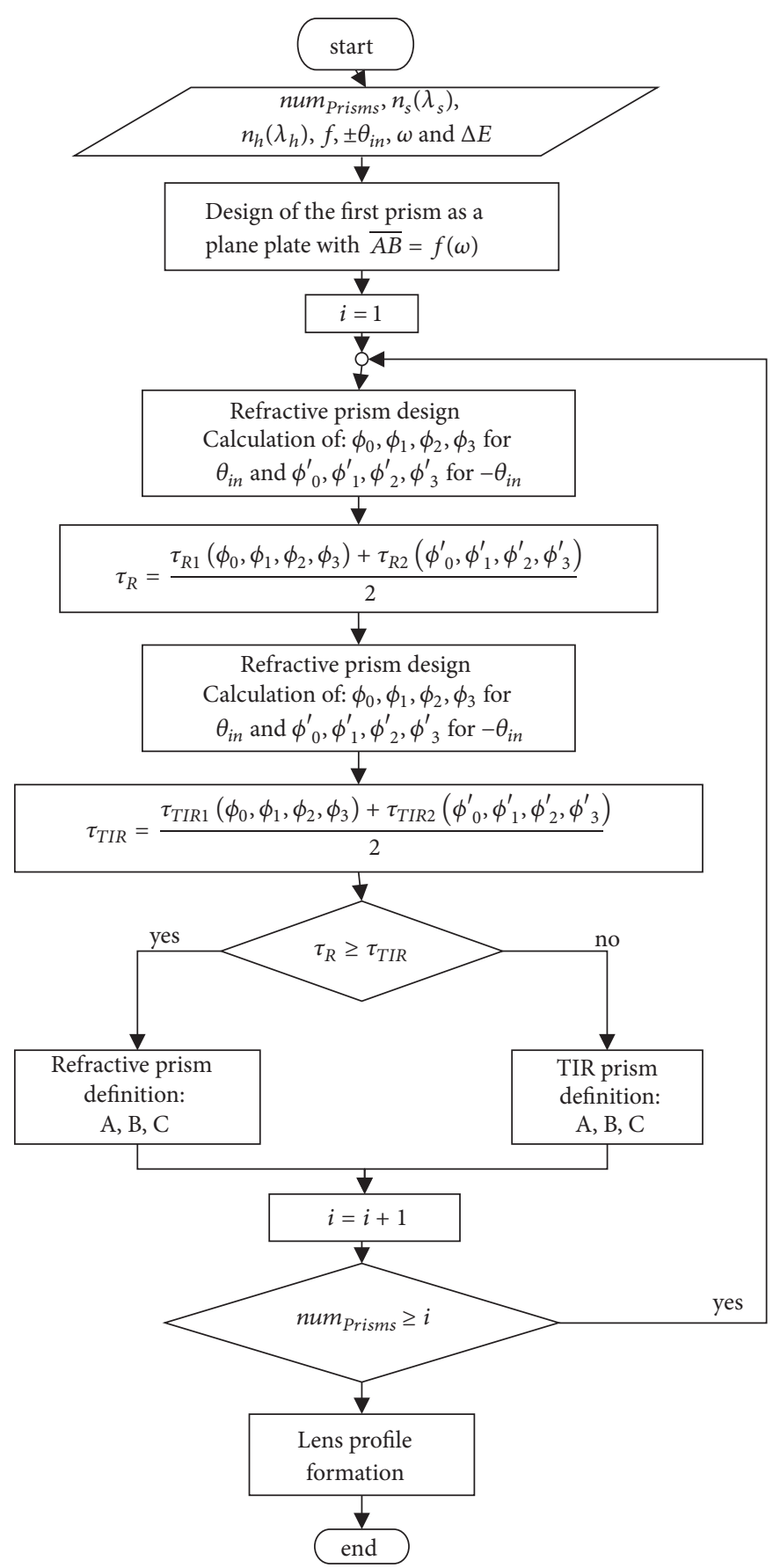

(b)

Figure 5: Nonimaging Fresnel lens design. (a) Design method for nonimaging Fresnel lenses composed of one kind of prisms; the equations used to solve $\beta$ are determined by the kind of prism to be tested. (b) Design method proposed, where the solution of each kind of prism corresponds to the design method shown in (a).

2.5. Hybrid Nonimaging Fresnel Lens Design Method. A summary of the design method for nonimaging Fresnel lenses is shown in Figure 5(a), where the solution of $\beta$ will be obtained by (1)-(5) for refractive prisms or by (7)-(11) for TIR prisms.

The new design method proposed is shown in Figure 5(b), where it is necessary to design both kinds of prisms for each iteration; the individual design of each kind of prism follows the algorithm shown in Figure 5(a). Then, the transmittance for the positive semiangle, $\tau_{1}\left(\theta_{\text {in }}\right)$, and for the negative semiangle, $\tau_{2}\left(-\theta_{\text {in }}\right)$, is computed taking into account (6) and the $\phi_{i}$ angles for each case. Finally, the highest average transmittance, $\tau_{R}$ or $\tau_{T I R}$, is selected to choose the best option of the prism. 


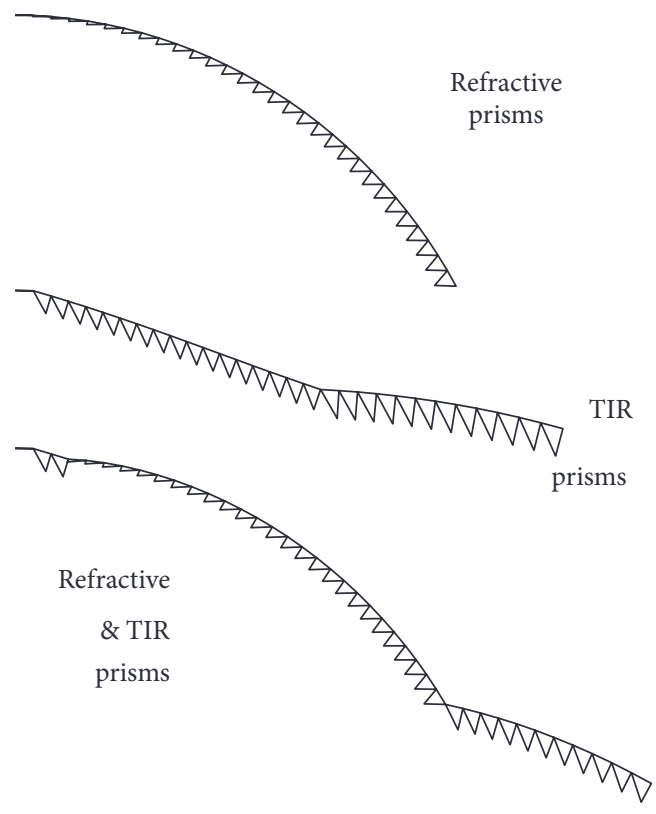

Figure 6: Profile of 3 different Fresnel lenses: one using only refractive prisms, another with TIR prisms, and a hybrid version with the 2 types of prisms.

\section{Results and Discussion}

Taking into account the design methods presented above, three nonimaging Fresnel lenses were designed with the objective to compare their transmittance along their profiles:

(1) Refractive nonimaging Fresnel lens: it is a chain of refractive prisms.

(2) TIR nonimaging Fresnel lens: it is integrated only by TIR prisms.

(3) Hybrid nonimaging Fresnel lens: before adding a new prism to the profile, the transmittance of a refractive prism and a TIR prism is calculated, and the one with the highest transmittance is added to the profile, looking for the losses reduction.

For all the cases, the input parameters were fixed to avoid unnecessary variations in the final results, and these parameters are shown in Table 1.

Figure 6 presents an illustrative profile of the Fresnel lens designed. The refractive Fresnel lens has a final surface with a curvature similar to a sphere, the TIR Fresnel lens has straight sections, and the hybrid lens is a combination of surfaces.

The transmittance of each prism considering the optical path for $\theta_{\text {in }}>0^{\circ}$ and $\theta_{\text {in }}<0^{\circ}$ was calculated and plotted as shown in Figures $7(\mathrm{a}), 7(\mathrm{~b})$, and $7(\mathrm{c})$. Also, the average transmittance of both optical paths for each prism was plotted. In Figure $7(\mathrm{~d})$, the average transmittance of every prism was compared. Figures 7(a) and 7(b) show that, after prisms 295 and 325, for the refractive and TIR cases, respectively, there is no convergence in the solution of the prism and, as a consequence, the limitation of the number of prisms composing the lens.
TABLE 1: Design parameters for all the nonimaging Fresnel lenses.

\begin{tabular}{lc}
\hline Wavelength range & $\lambda_{s}=390 \& \lambda_{h} 750 \mathrm{~nm}$. \\
\hline Refractive index & PMMA (See Eq. (12)) \\
\hline Focal distance & $50 \mathrm{~cm}$. \\
\hline Number of prims num $_{\text {Prisms }}$ & 325 \\
\hline$\pm \theta_{\text {in }}$ & $25^{\circ}$ \\
\hline$\omega$ & $0.2^{\circ}$ \\
\hline$\Delta E$ & 0.0001 \\
\hline
\end{tabular}

The design method presented allows the design of nonimaging Fresnel lenses with higher efficiency; this is accomplished due to reducing the losses related to the reflectance at the refractive surface. As can be seen in Figure 7, for the hybrid lenses, it is possible to modulate transmittance; when it starts to decrease, it is forced to increase it, keeping it nearly constant. This result is not possible with other lenses.

The space distribution profile for each lens is shown in Figure 8 and its average efficiency was calculated as

$$
\varepsilon=\frac{\Phi_{\text {in }}}{\Phi_{\text {out }}}
$$

where $\Phi_{\text {in }}$ is the amount of light inciding into the lens and $\Phi_{\text {out }}$ is the amount of light collected inside the focal area that is represented by the dotted-red line. As the lenses offered a range of acceptance angles from $-\theta_{\text {in }}$ to $\theta_{\text {in }}$, the final efficiency shown in Figure 8 includes two sources, one with an incident angle $\theta_{i n}$ and the other with $-\theta_{i n}$.

Considering the mathematical analysis done, the refractive Fresnel lens (Figure 8(a)) presents a defined collection area with an average efficiency of $70 \%$, which means that all the collected light for the range from $-\theta_{\text {in }}$ to $\theta_{\text {in }}$ is redirected to the circular surface. In contrast, the TIR Fresnel lens (Figure $8(\mathrm{~b})$ ) has a transmittance of $77.1 \%$ but a portion of the collected light arrives outside the collection area, having a final efficiency around 55.1\%. Moreover, the hybrid Fresnel lens (Figure 8(c)), as in the case of the refractive lens, has an average transmittance of $90.1 \%$, where all the light is collected, inside the collection area, having a final efficiency (85\%) similar to the transmittance calculated.

The aberration in space distribution for the TIR lenses is presented due to the rotational symmetry applied over prism with sides $A C$ and $B C$ higher than $A B$, as can be seen in Figure 4, which leads to the shading over next prism in the inner half of the lens. In the case of the hybrid lens, this shading effect is avoided because, as can be seen in Figure 6, the orientation of the prisms changes in the outer prism.

\section{Conclusions}

The design method proposed is a variation to the design method presented by Leutz and includes the proposal made by Akisawa to reduce the losses related to the chromatic aberration. In the example shown in the previous section, the hybrid lens prevents transmittance decreasing and allows the lens to maintain its efficiency and collection area, reducing the aberrations. In contrast, this new design presents some 


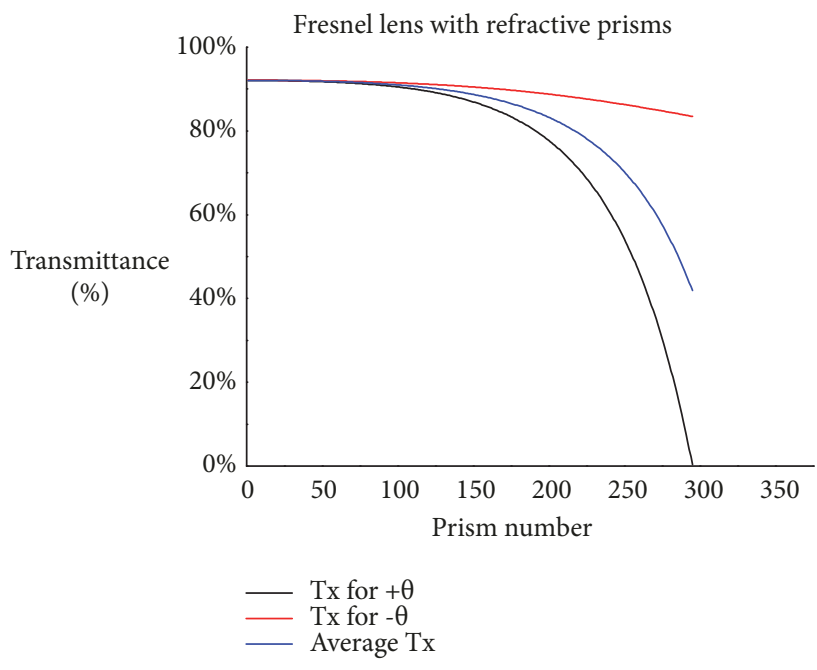

(a)

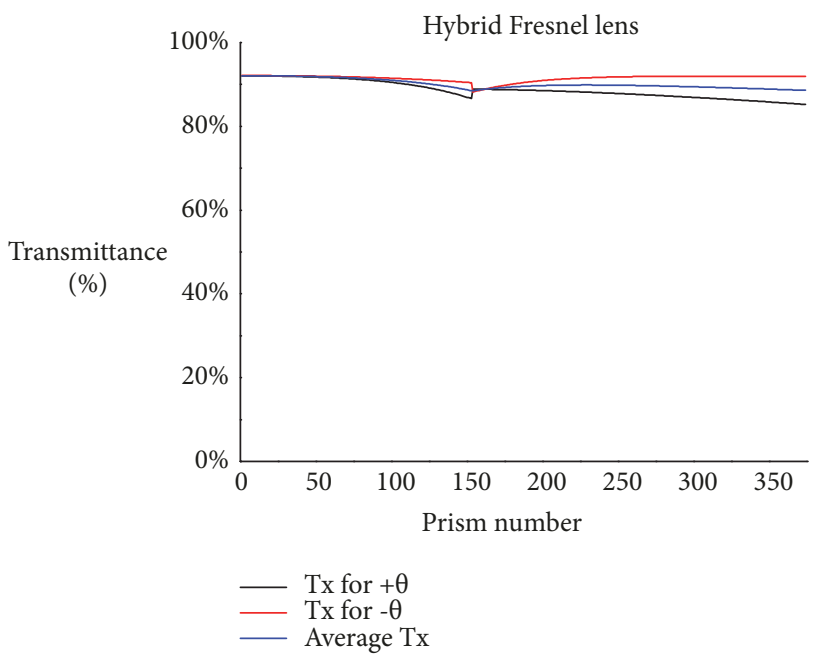

(c)

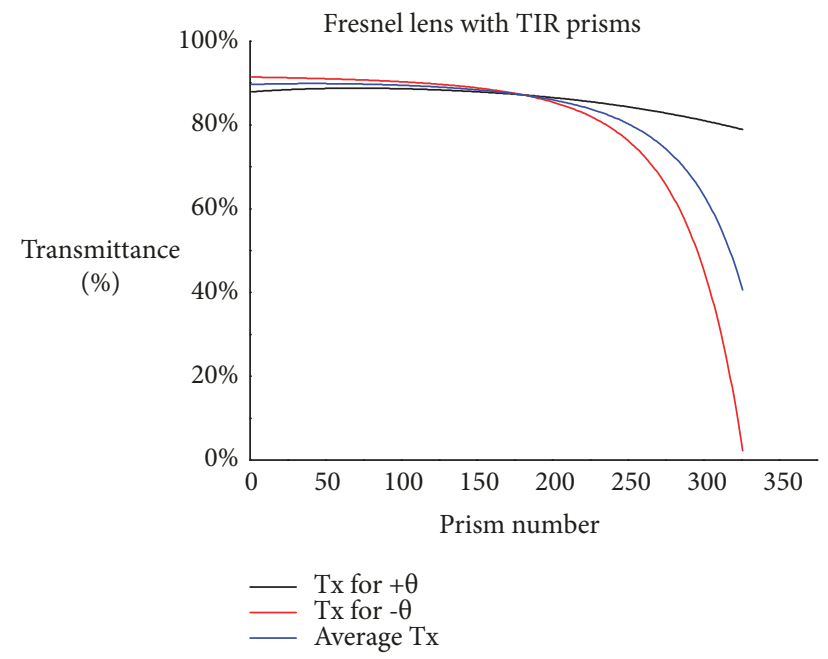

(b)

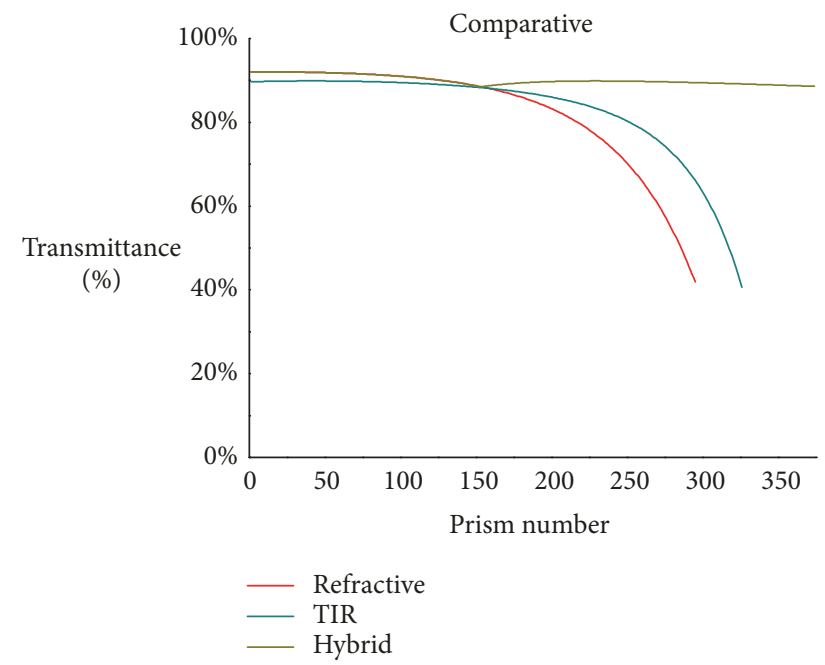

(d)

FIGURE 7: Transmittance obtained in each of the prisms of 3 different Fresnel lenses with the same input parameters. The black line in graphs (a), (b), and (c) corresponds to the transmittance for the optical path when $\theta_{\text {in }}>0^{\circ}$ and the red line when $\theta_{\text {in }}<0^{\circ}$, and the blue line is the average of both. The graph is a Fresnel lens with refractive prisms, (b) is a Fresnel lens with TIR prisms, and (c) is a hybrid Fresnel lens. Graph (d) is a comparison between the average transmittance of the 3 lenses.

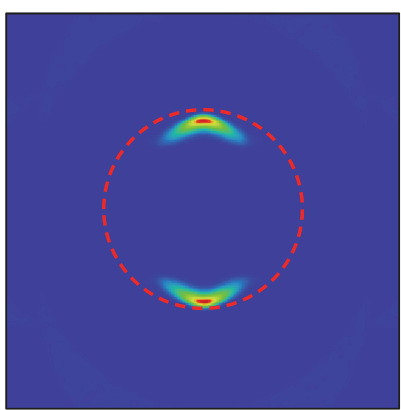

(a) Efficiency: $70 \%$

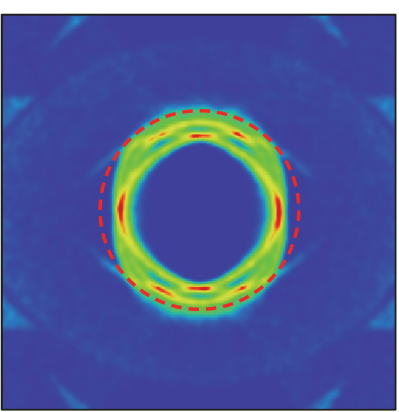

(b) Efficiency: $55 \%$

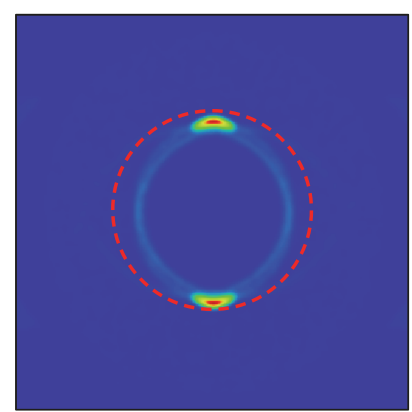

(c) Efficiency: $85 \%$

FIgURE 8: Space distribution profile for (a) refractive, (b) TIR, and (c) hybrid nonimaging Fresnel lenses for the extreme values of $\theta_{\text {in }}$. The dotted red line represents the collection area for each case and the efficiency is the rate of light collected inside this area in relation to the amount of incident light over the lens. 
disadvantages as the problems related to the manufacture and cleaning process because the lens is no longer a smooth surface.

The differences between the transmittance and the final efficiencies are related to the aberrations of the spatial distribution and the absorption losses.

For future work, an extensive mathematical and numerical analysis of the transmittance and efficiency will be presented, for example, including polarized light in our models and also including novel techniques for avoiding losses and aberrations.

\section{Data Availability}

The data used to support the findings of this study are available from the corresponding author upon request. Besides, the individual transmittances for the lenses designed are included within the supplementary information file.

\section{Conflicts of Interest}

The authors declare that they have no conflicts of interest.

\section{Acknowledgments}

This work was supported by Universidad Autónoma de Nuevo León and by the National Council of Science and Technology (CONACyT) under grant 387533.

\section{Supplementary Materials}

The individual transmittances for the three lenses designed are presented in a data sheet added as supplementary materials. (Supplementary Materials)

\section{References}

[1] R. Leutz and A. Suzuki, in Nonimaging Fresnel Lenses, vol. 83 of Physics and Astronomy online library, Springer, Berlin, Germany, 2001.

[2] W. T. Xie, Y. J. Dai, R. Z. Wang, and K. Sumathy, "Concentrated solar energy applications using Fresnel lenses: a review," Renewable \& Sustainable Energy Reviews, vol. 15, no. 6, pp. 2588-2606, 2011.

[3] I. Wallhead, T. M. Jiménez, J. V. G. Ortiz, I. G. Toledo, and C. G. Toledo, "Design of an efficient Fresnel-type lens utilizing double total internal reflection for solar energy collection," Optics Express, vol. 20, no. 106, pp. A1005-A1010, 2012.

[4] Z. Zhuang and F. Yu, "Optimization design of hybrid Fresnelbased concentrator for generating uniformity irradiance with the broad solar spectrum," Optics \& Laser Technology, vol. 60, pp. 27-33, 2014.

[5] A. Akisawa, M. Hiramatsu, and K. Ozaki, "Design of domeshaped non-imaging Fresnel lenses taking chromatic aberration into account," Solar Energy, vol. 86, no. 3, pp. 877-885, 2012.

[6] F. A. Jenkins, H. E. White, and D. G. Brukhard, "Fundamentals of Optics," American Journal of Physics, vol. 26, no. 4, p. 272, 1958. 


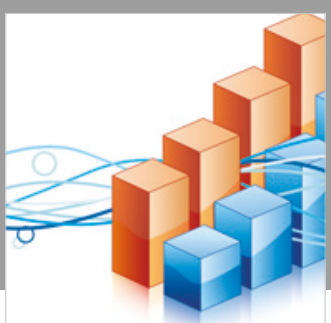

Advances in

Operations Research

\section{-n-m}
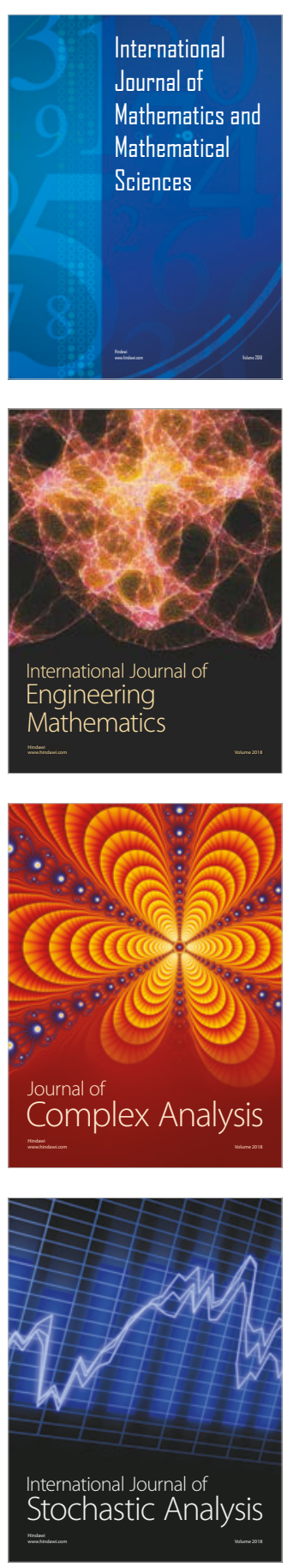
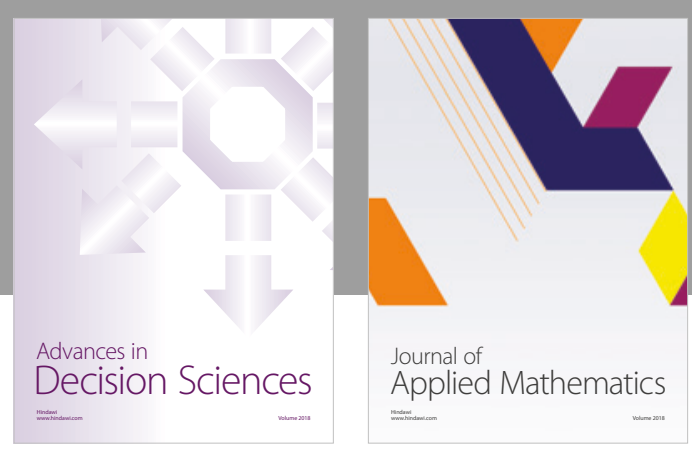

Journal of

Applied Mathematics
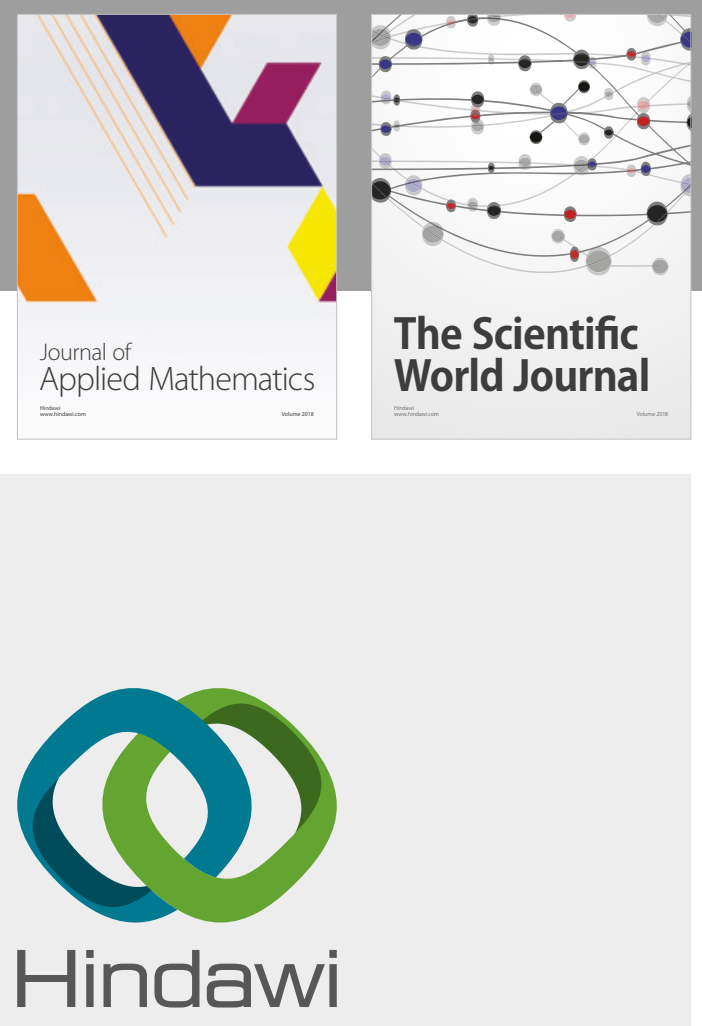

Submit your manuscripts at

www.hindawi.com

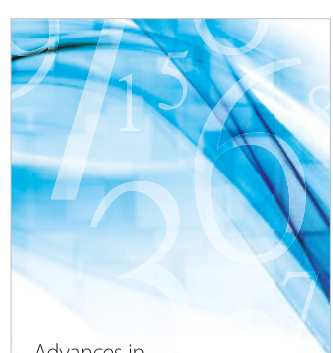

Advances in
Numerical Analysis
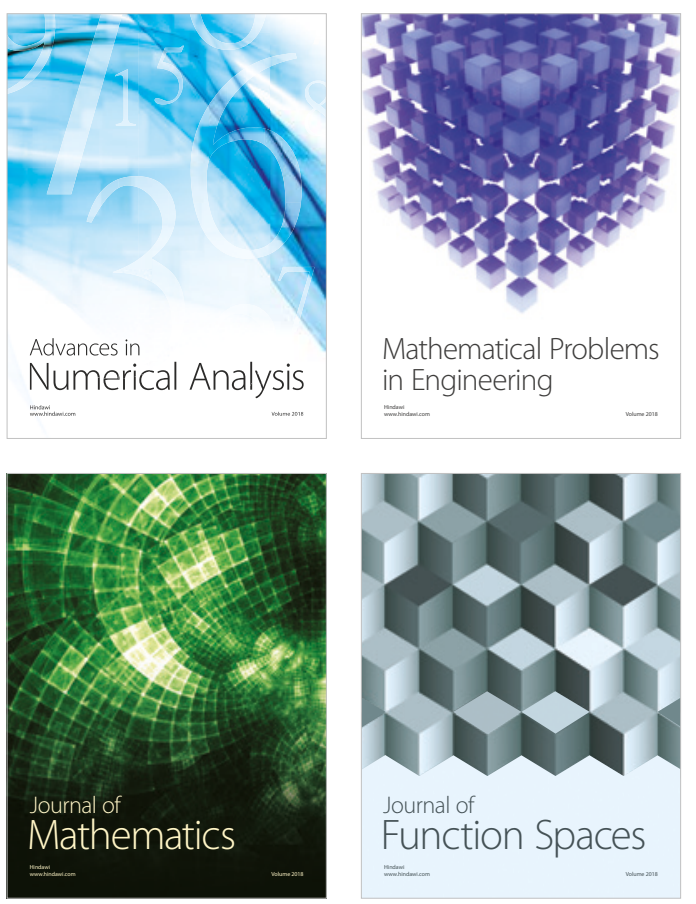

Mathematical Problems in Engineering

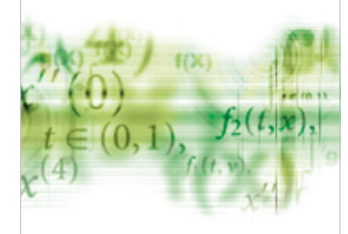

International Journal of

Differential Equations

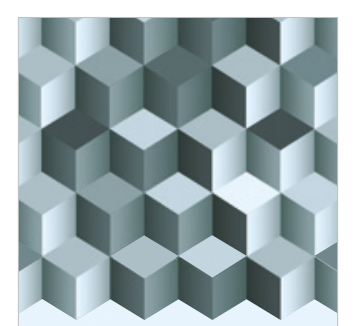

Journal of

Function Spaces

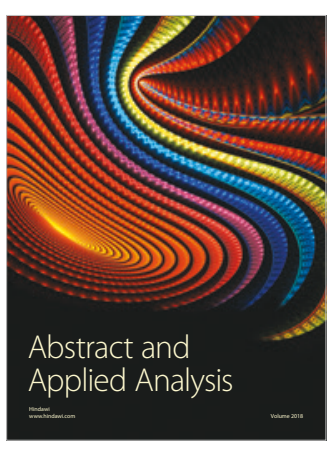

The Scientific

World Journal

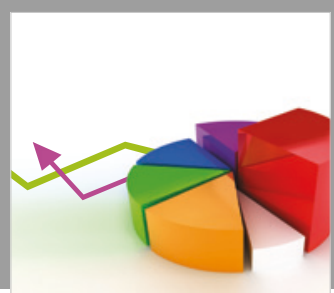

Journal of

Probability and Statistics
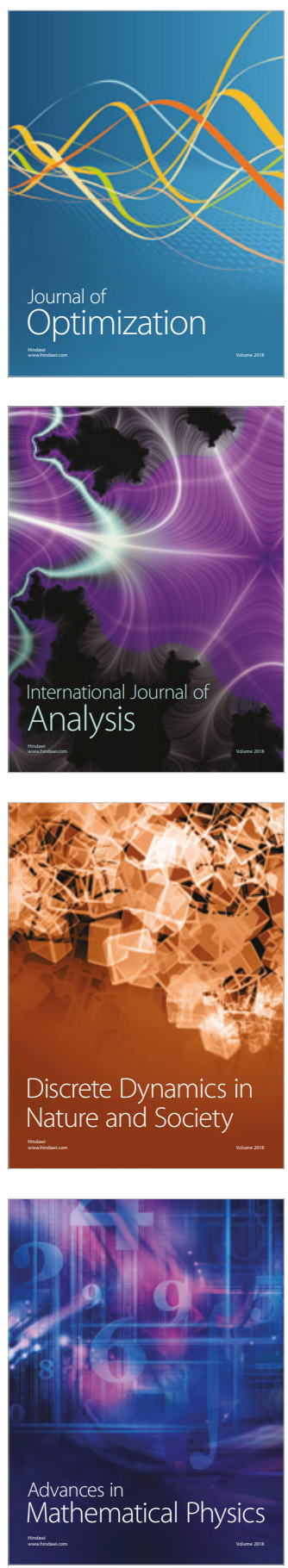